\section{Nano-Imprint Mold and Direct Imprinting Technique Creates Single Sub-20-Nanometer-Wide, Centimeter-Long Nanofluidic Channel}

Many leading-edge applications in biochemical sensors and microfluidics require isolated, continuous fluidic channels, with lengths on the order of a cm, and widths on a $20-n m$ scale. Traditional nanolithographic tools are challenged by the strict limits on line edge roughness (to prevent clogging) over the desired centimeter-long channel length, which is much larger than typical writing fields. X. Liang and colleagues at Princeton University have demonstrated a novel direct imprinting technique to fabricate a single fluidic channel of uniform width (11-50 nm) and over $1.5 \mathrm{~cm}$ long.

As reported in the December 2007 issue of Nano Letters (p. 3774; DOI: $10.1021 / \mathrm{nl072253x}$ ), the researchers first created the nano-imprint mold. They grew about $10 \mathrm{~nm}$ of $\mathrm{SiO}_{2}$ on a (110)oriented silicon-on-insulator wafer, then lithographically patterned a $0.5 \mathrm{~cm} \times 1.5 \mathrm{~cm}$ rectangle in the $\mathrm{SiO}_{2}$. The long edge was aligned to the $\{111\}$ crystallographic axis in Si. An anisotropic KOH-based etch of the silicon, using the silicon dioxide as a mask and the underlying insulator as a stop layer, created a mesa with vertical sidewalls of $\{111\}$ planes. The much slower etching rate in the $<111>$ directions versus the $<110\rangle$ directions ensured that the sidewalls were nearly atomically smooth, despite the severe edge roughness of the original etch mask. Subsequently, the $\mathrm{SiO}_{2}$ was stripped away. A thin layer of $\mathrm{Si}_{x} \mathrm{~N}_{y}$ was then deposited uniformly and conformally, by low-pressure chemical vapor deposition, over the entire wafer surface including the sidewalls. The thickness of this film defined the channel width. An anisotropic reactive ion etch (RIE), acting in the vertical direction, removed the $\mathrm{Si}_{x} \mathrm{~N}_{y}$ on all horizontal surfaces while leaving some on the relatively tall sidewalls. By selectively etching away the remnants of the Si mesa, the long "fin" of $\mathrm{Si}_{x} \mathrm{~N}_{y}$ was isolated and became the protrusion of an imprint mold.

During the imprinting step, the mold was pressed into a UV-curable material layer. The resulting nanochannel could be used directly or as an etching mask to transfer the channel into an underlying substrate (e.g., $\mathrm{SiO}_{2}$ ) using RIE.

The researchers characterized the channel dimensions using scanning electron microscopy and completed a nanofluidic device by fabricating a pair of liquid reservoirs at each end. They passed water containing a fluorescent marker through the nanochannel and also flowed DNA in solution (which stretched as it passed into and through the device).

"This approach to creating and precisely placing a single long, narrow and continuous nanofluidic channel removes a key obstacle to developing many innovative biological and chemical sensors such as single-strand DNA sequencers," Liang said.

Rich LOUIE

\section{Metallodielectric Multilayer Stacks Show Enhanced Ultrafast Optical Nonlinear Response}

In the December 1, 2007 issue of Optics Letters (p. 3435), G. Ma and S.H. Tang of Shanghai University in China and the National University of Singapore, respectively, reported the enhancement of the nonlinear optical response in $\mathrm{Ag}-\mathrm{TiO}_{2}$ multilayer stacks determined through femtosecond optical Kerr effect measurements.

\section{Correction}

The volume number for the December 2007 issue of MRS Bulletin was incorrectly printed on the cover as $\mathbf{3 4}$. The correct volume number is $\mathbf{3 2}$.

\section{Advertisers in This Issue}

Page No.

\begin{tabular}{lr}
\hline High Voltage Engineering Europa B.V. & Inside front cover \\
\hline Huntington Mechanical Laboratories, Inc. & Outside back cover \\
\hline Janis Research Company, Inc. & 6 \\
\hline MMR Technologies, Inc. & 6 \\
\hline National Electrostatics Corp. & 20 \\
\hline Society of Vacuum Coaters & 26 \\
\hline
\end{tabular}

For free information about the products and services offered in this issue, check http://www.mrs.org/bulletin_ads.
Nonlinear materials with large thirdorder nonlinear susceptibility, $\chi^{(3)}$, and ultrafast response are key elements in developing ultrafast all-optical switches. In the past decade, researchers have studied metallic nanoparticles embedded in dielectric matrices. However, these composite materials have exhibited a common drawback: the close association of a large $\chi^{(3)}$ with a large linear absorption, which in turn leads to large propagation losses and might induce thermal damage of the potential device.

In recent years researchers have shown that metal dielectric multilayer structures can show a high transmission in specific regions of the spectrum due to multiple Bragg reflections, even when the total metal thickness significantly exceeds the conventional skin depth. In this work, the researchers prepared $\mathrm{Ag}-\mathrm{TiO}_{2}$ multilayer stacks by using alternate sputter deposition. By selecting suitable thicknesses for the metal and dielectric layers, they have designed a $\mathrm{Ag}-\mathrm{TiO}_{2}$ structure with a maximum transmission of about $35 \%$ at $800 \mathrm{~nm}$, even though the transmission of a similar thickness of pure $\mathrm{Ag}$ film is $<1.5 \%$. The researchers performed femtosecond optical Kerr measurements at $800 \mathrm{~nm}$ using Ti:sapphire laser pulses with a temperal duration of $120 \mathrm{fs}$ and repetition rate of $82 \mathrm{MHz}$. The results show that the optical nonlinearity of the multilayer structure can be as large as $3.2 \times 10^{-9} \mathrm{esu}$, which is much larger than that shown by a pure 25-nm-thick Ag film studied for comparison $\left(1.1 \times 10^{-9} \mathrm{esu}\right)$. By using the matrix transfer method the researchers showed that the amplitude of the electric field within the Ag metal layers was clearly larger within the metal layers in the metaldielectric multilayer structure than in pure metal films. The researchers concluded that the origin of the large nonlinearity arose from the fact that the light can penetrate the intrinsically high nonlinear metallic layers due to the specially designed metal-dielectric structure. They said that these results demonstrate that metal-dielectric multilayer composites are suitable candidates for fabricating alloptical switching photonic devices.

ROSALÍA SERNA

\section{Laser Control of Alignment and Rotation of Polyatomic Molecules Demonstrated}

The reactivity of molecules is strongly correlated to their spatial orientation, and the ability to manipulate that orientation can enhance applications such as ultrafast $\mathrm{x}$-ray imaging and time-resolved photoelectron spectroscopy. S.S. Viftrup at the University of Aarhus in Denmark, 
S. Trippel of the University of Freiburg in Germany, E. Hamilton at Northwestern University, and their colleagues have reported in the October 5, 2007 issue of Physical Review Letters (DOI:10.1103/ PhysRevLett.99.143602) an effective method to control the alignment and chronized nanosecond and femtosecond laser pulses. The researchers also used quantum mechanical simulations to support their experimental findings on the efficacy of their method to align and manipulate molecules.

Laser alignment of molecules results rotation of molecular objects using syn-

from the interaction of the laser pulse with the dipole moment of the molecule. The research team employed a 10 nanosecond duration laser pulse to align molecules of 3,5 difluoroiodobenzene (DFIB) along its most polarizable axis (the axis defined by the bond between the $\mathrm{C}$ and I atoms). They then applied an orthogonally polarized 150 femtosecond duration pulse to activate motion about the aligned axis, resulting in rotation. The orientation and movement of the molecules was measured by monitoring ion fragments released from the molecules through pulsed excitation, as shown in Figure 1. By varying the length and
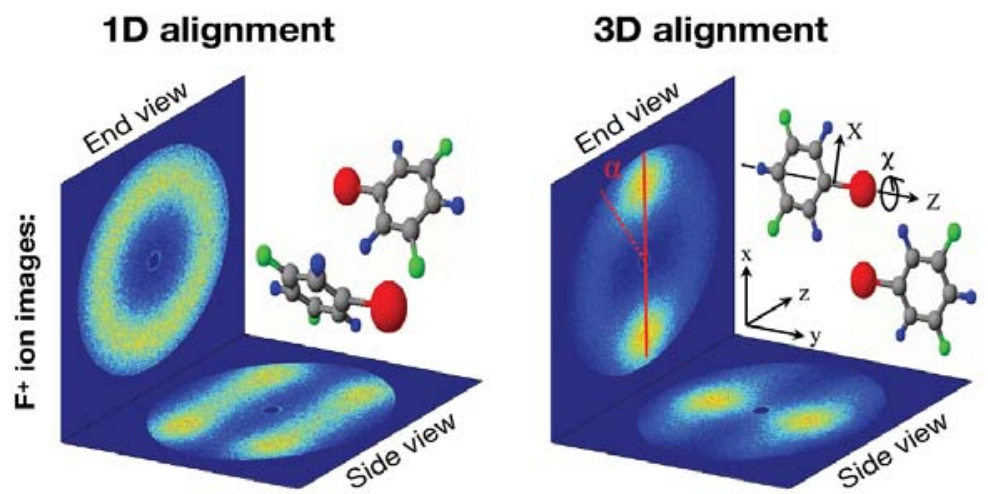

b
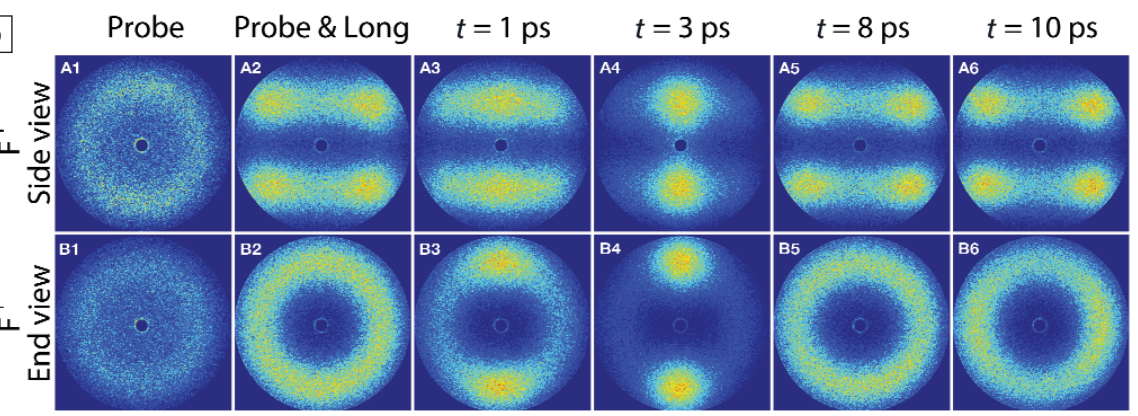

C

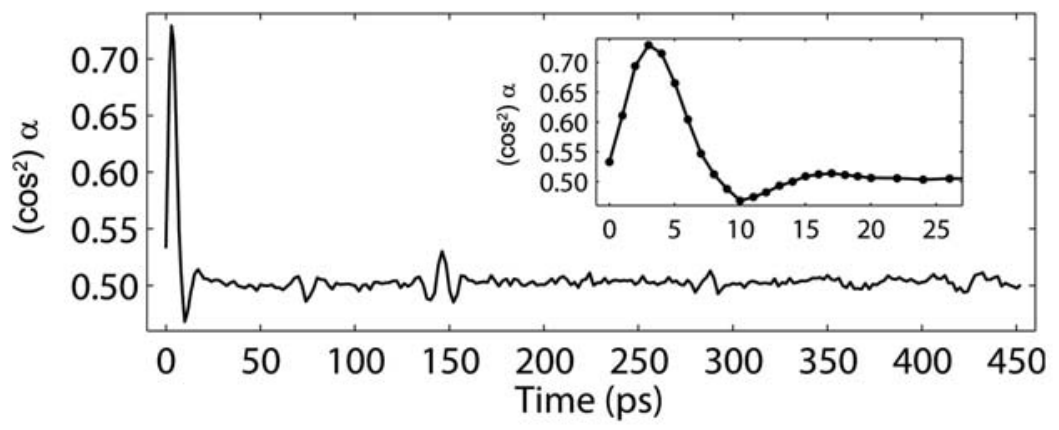

Figure 1: An overview of the laser pulse molecule alignment technique. (a) $\mathrm{F}^{+}$ion imaging of 3,5 difluoroiodobenzene (DFIB) shows alignment in one and three dimensions, respectively, with the coordinate system shown in red. (b) Time-lapsed $\mathrm{F}^{+}$ion imaging of DFIB shows the dephasing and revival of 3D alignment. (c) Stimulated rotation of the molecule, as measured by the expectation value of the angle of rotation about the tightly aligned molecular axis $\left(\cos ^{2}\right) \alpha$, shown as a function of time after the short pulse. Reprinted with permission from Physical Review Letters 99 (2007) 143602. @2007 by the American Physical Society. strength of the nanosecond and femtosecond alignment pulses, the researchers uncovered a parameter space within which the behavior of the molecule could be controlled. A numerical solution based on a nonperturbative solution of the Schrödinger equation of the molecular dipole and two laser fields provided a quantum mechanical basis for describing the behavior of the molecule.

Though these experiments were carried out on 3,5 difluoroiodobenzene, the researchers generalized their model for an arbitrary molecular system. Furthermore, they expect, based on their numerical calculations, that by applying more sophisticated pulse sequences to the molecule, field-free alignment will be achieved. This method could prove fruitful for investigations exploring the rotational dynamics of complex molecules as well as for control of molecular torsions.

ARTHUR FELDMAN

\section{Cooperative Conduction \\ Mechanisms Determined for Candidate Fuel Cell Electrolyte}

Fuel cells are gaining increased attention recently as an efficient and clean method of generating electricity. An example is the solid-oxide fuel cell, where solid oxide refers primarily to the materials used for the electrolyte, and good conduction paths for $\mathrm{O}^{2-}$ or $\mathrm{H}^{+}$ions are required. Optimizing the conductivity in the electrolyte can significantly increase the overall efficiency of the cell. The novel oxide $\mathrm{La}_{1-x} \mathrm{Ba}_{1+x} \mathrm{GaO}_{4-x / 2}$ is a promising candidate electrolyte in the emerging class of proton-conducting ceramics, due to its intermediate operating temperature, $500-700^{\circ} \mathrm{C}$, and the high conductivities of both oxygen ions and protons. E. Kendrick and P.R. Slater of the University of Surrey, J. Kendrick of the University of Bradford, K.S. Knight of CCLRC Rutherford Appleton Laboratory and the Natural History Museum, and M.S. Islam of the University of Bath have determined the unusual conduction mechanisms in this system through a combined experimental and computational approach, a vital step toward optimizing the conductivity. They published their findings in the November 2007 issue of Nature Materials (p. 871, DOI:10.1038/nmat2039).

Unlike the perovskite and fluorite compounds typically used in solid-oxide fuel cells, $\mathrm{La}_{1-x} \mathrm{Ba}_{1+x} \mathrm{GaO}_{4-x / 2}$ has tetrahedral structural units, where the Ga atom is surrounded by a group of four oxygen ions in a tetrahedral configuration. The researchers found that this structure is key to the mechanism by which oxide ions move through the lattice. They discovered that an oxygen vacancy is accommodated 